\title{
All-optical image processing with cavity type II second-harmonic generation
}

\author{
Pierre Scotto, Pere Colet, and Maxi San Miguel
}

Instituto Mediterráneo de Estudios Avanzados, Consejo Superior de Investigaciones Científicas, Universitat de les Illes Balears, E-07071 Palma de Mallorca, Spain

Received March 4, 2003

\begin{abstract}
The possibilities offered by type II intracavity second-harmonic generation for all-optical parallel processing of images are investigated. By injecting an image in a linearly polarized pump beam and a homogeneous field with orthogonal polarization, we obtain, according to the value of the latter, either frequency and polarization transfer or contrast enhancement and contour recognition. Noise-filtering effects are also predicted. (C) 2003 Optical Society of America
\end{abstract}

OCIS codes: $190.0190,190.4420,190.1450,190.4410,100.0100$.

In this Letter we explore the possibilities offered by intracavity type II second-harmonic generation (SHG) for all-optical image processing. ${ }^{1-4}$ The system we consider is a crystal with a $\chi^{(2)}$ nonlinearity enclosed in an optical cavity, taken ideally to be a planar cavity. Assuming type II phase matching, a second-harmonic (SH) field will be generated if the cavity is pumped at two orthogonal polarizations $x$ and $y$. The possibility of independently tailoring the pump field at each polarization, combined with threshold effects characteristic of nonlinear dynamics in a cavity, make this system particularly interesting. To demonstrate, we consider the nonlinearly coupled mean field equations, ${ }^{5-8}$

$$
\begin{gathered}
\partial_{t} B=-\left(1+i \Delta_{0}\right) B+\frac{i}{2} \nabla_{\perp}^{2} B+i A_{x} A_{y}, \\
\partial_{t} A_{x}=-(1+i \Delta) A_{x}+i \nabla_{\perp}^{2} A_{x}+i A_{y}^{*} B+E_{x}, \\
\partial_{t} A_{y}=-(1+i \Delta) A_{y}+i \nabla_{\perp}^{2} A_{y}+i A_{x}^{*} B+E_{y},
\end{gathered}
$$

which govern the temporal evolution of the intracavity field envelopes $A_{x}$ and $A_{y}$ at the fundamental frequency with polarization $x$ and $y$ and $B$ at the $\mathrm{SH}$ frequency, polarized along the $y$ axis. $\Delta_{0}(\Delta)$ is the detuning at the SH (fundamental) frequency. Time and lengths are expressed in units of the cavity decay time and diffraction length, respectively, with diffraction coming into play through the transverse Laplacian $\nabla_{\perp}^{2}$. The pumping amplitudes $E_{x}$ and $E_{y}$ in each linear polarization state are chosen such that the image to be processed is injected with the $x$ polarization, whereas $E_{y}$ is a homogeneous field.

Study of the steady-state solution of Eqs. (1)-(3) for homogeneous pumps provides valuable insight into the properties of the system. By fixing $E_{y}$ to a given value ${ }^{9}$ the typical dependence of the intracavity fields $A_{x}, A_{y}$, and $B$ on $E_{x}$ (Fig. 1) can be summarized as follows. For small $E_{x}$ the function $A_{x}\left(E_{x}\right)$ is single valued and takes small values of $\sim E_{x}$, whereas $A_{y}\left(E_{x}\right)$ is large and close to $E_{y} /(1+i \Delta)$, which is obtained from Eqs. (1)-(3) for $E_{x}=0$. For $E_{x}$ close to $E_{y}$ both functions $A_{x}\left(E_{x}\right)$ and $A_{y}\left(E_{x}\right)$ become $\mathrm{S}$ shaped; therefore the system displays bistability. Above a certain value of $E_{x}$ larger than $E_{y}, A_{x}\left(E_{x}\right)$ and $A_{y}\left(E_{x}\right)$ are again single valued, but $A_{x}\left(E_{x}\right) \gg A_{y}\left(E_{x}\right)$. The existence of three steady-state solutions of Eqs. (1)-(3) in a region of finite width centered on $E_{x}=E_{y}$ is closely related to the polarization instability occurring in the symmetrical pumping case $E_{x}=E_{y}=E$ for a pump above the critical value $e^{5-8,10}$

$$
E_{\text {as }}^{2}=2\left(1+\Delta_{0}^{2}\right)^{1 / 2}\left(1+\Delta^{2}\right)^{3 / 2}+2\left(1+\Delta^{2}\right)\left(1-\Delta \Delta_{0}\right) .
$$

In fact, this S shape can be observed only if $E_{y}>E_{\text {as }}$.

We now consider the injection of an image; that is, the amplitude of the ( $x$-polarized) signal $E_{x}(x)$ is a function of the transverse coordinate $x$. At a given position $x$ the intracavity fields $A_{x, y}(x)$ and $B(x)$ tend to take the stationary values shown in Fig. 1 as if the pumps were homogeneous, despite the spatial coupling caused by diffraction. Diffraction becomes relevant for image details on the scale of the diffraction length. If $E_{x}(x)$ remains well below $E_{y}, A_{x}(x)$ never leaves the lower branch of the curve $A_{x}\left(E_{x}\right)$ (Fig. 1), and the output at the SH frequency $B(x)$ reproduces the spatial distribution of the input signal $E_{x}(x)$. This is illustrated in Fig. 2 for the one-dimensional case and is corroborated in two dimensions (Fig. 3). Therefore intracavity type II SHG allows an input image to be transferred from the fundamental to the $\mathrm{SH}$ frequency.



(a)



(b)
Fig. 1. SHG for asymmetric pumping. Steady-state intracavity field amplitudes as a function of $E_{x}$ for $E_{y}=5$. $\Delta_{0}=0, \Delta_{x}=\Delta_{y}=1$. The symbols indicate the regions of the curves that are unstable, as predicted by a linear stability analysis. The vertical dashed line corresponds to the symmetric pumping $E_{x}=E_{y}$.

(C) 2003 Optical Society of America 




(a)



(b)

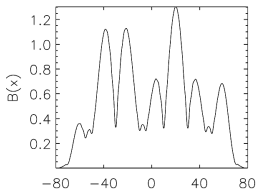

(c)
Fig. 2. (a) Input image (one-dimensional case) together with the homogeneous pump $E_{y}$ (dotted line). (b) $A_{x}(x)$. (c) $B(x)$ (frequency transfer).
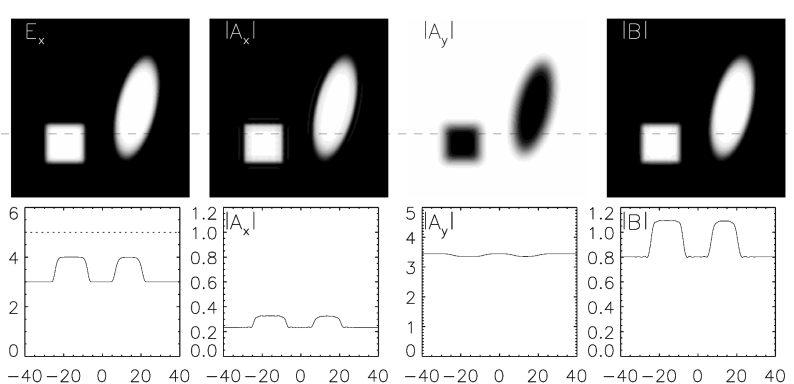

Fig. 3. Frequency transfer (two-dimensional case). Upper panels, spatial distribution of (from the left to the right) pump field $E_{x}$ (input image), $A_{x}, A_{y}$, and $B$, after the transients have disappeared. Lower panels, transverse cut of the upper panels along the horizontal dashed line. Dotted line in left panel, $E_{y}=5$.

In addition, polarization switching is performed, since the image encoded in $E_{x}$ and the $\mathrm{SH}$ field $B$ have orthogonal polarizations. As a side effect, the input image appears in negative as a weak modulation of $A_{y}$ around $E_{y} /(1+i \Delta)$.

If the amplitude of the signal locally exceeds $E_{y}$, the S-shaped dependence of $A_{x}\left(E_{x}\right)$ and $A_{y}\left(E_{x}\right)$ comes into play. At least when $E_{x}(x)$ crosses the limit of the hysteresis cycle (Fig. 1 ), $A_{x}\left[E_{x}(x)\right]$ will be forced to jump to the upper branch, giving rise to nearly discontinuous spatial variations of $A_{x}$ and $A_{y}$. In fact, we found numerically that this jump already occurs if the weaker condition $E_{x}(x)>E_{y}$ is fulfilled. This can be traced back to the fact that the two asymmetric solutions ( $A_{x}$ small, $A_{y}$ large $)$ and ( $A_{x}$ large, $A_{y}$ small) of Eqs. (1)-(3) are only equivalent for $E_{x}=E_{y}$. If $E_{x}=E_{y}-\epsilon(\epsilon>0)$ locally, the system, with vanishing intracavity fields as initial conditions, selects at that point the solution with ( $A_{x}$ small, $A_{y}$ large). For locations where $E_{x}=E_{y}+\epsilon$ the other solution is selected. A simple geometric construction (Fig. 4) allows us to predict the behavior of the system for a given input signal that eventually crosses the symmetrical point $E_{x}=E_{y}$. Figure 5 shows the results for a one-dimensional image. In the region where $E_{x}(x)$ exceeds $E_{y}, A_{x}(x)$ shows a large peak, magnifying the contrast between $E_{x}$ and $E_{y}$. Elsewhere, the image is very much attenuated. By lowering the value of the homogeneous pump $E_{y}$, a larger part of the figure is amplified, as shown in Fig. 6 . In fact, changing $E_{y}$ allows us to scan the image fully. At the crossing points $E_{x}=E_{y}$ the SH field $B(x)$ displays a sharp peak, since $A_{x}(x) \simeq A_{y}(x)$ locally; i.e., the system goes through the symmetric steady-state solution characterized by a higher intracavity SH field than the asymmetric stable ones. ${ }^{5}$ As a consequence, in two dimensions (Fig. 7) the $\mathrm{SH}$ field clearly displays the contour of the image at the field level fixed by the pump $E_{y}$, whereas $A_{x}$ reproduces the input image with much better contrast. Simultaneously, the image appears in negative in the orthogonal polarization $A_{y}$ with comparable contrast. It can be seen from Fig. 7 that image processing is slightly affected by diffraction effects in two dimensions, which tends to smooth out sharp angles in the



Fig. 4. Geometrical construction used to predict the behavior of the system to a given input signal. The case considered here corresponds to contrast enhancement and contour recognition.



(a)

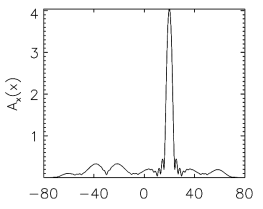

(b)

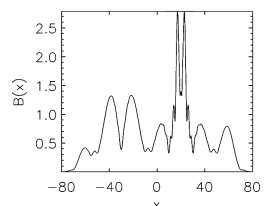

(c)
Fig. 5. (a) Input signal $E_{x}(x)$ (one-dimensional case) and $E_{y}=6$ (dotted line). (b) $A_{x}(x)$ (contrast enhancement). (c) $B(x)$ (contour recognition).

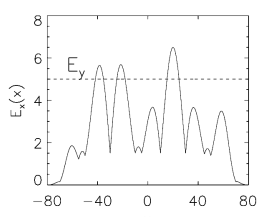

(a)

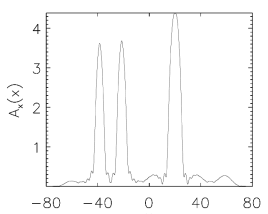

(b)



(c)
Fig. 6. Same as Fig. 5 but with $E_{y}=5$.

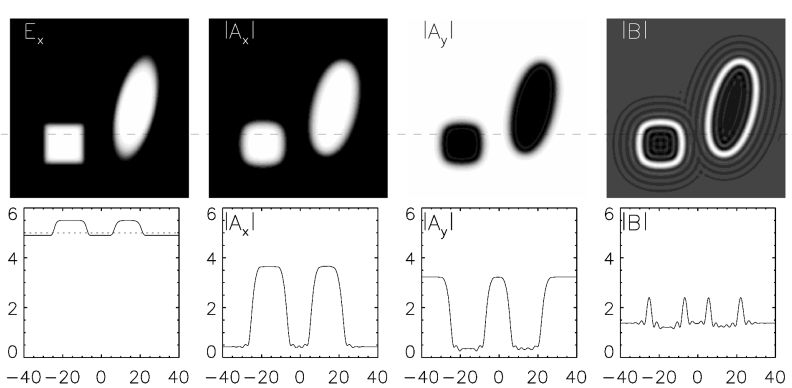

Fig. 7. Contrast enhancement and contour recognition in the noiseless case. 

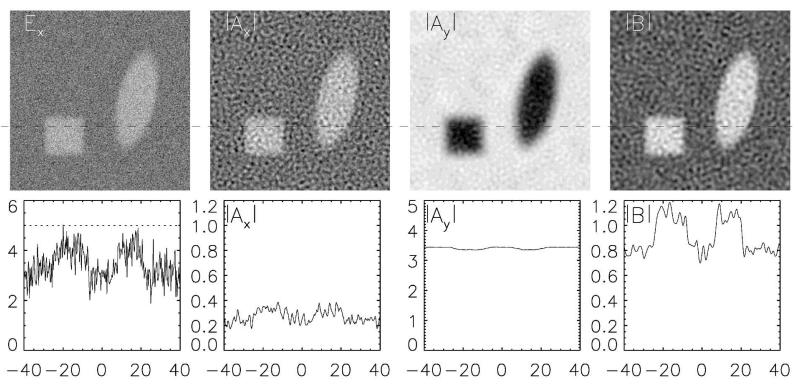

Fig. 8. Frequency transfer of a two-dimensional optical image in the noisy case and noise filtering.

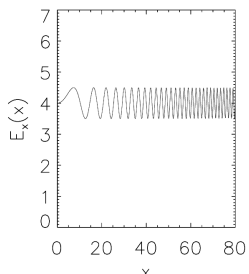

(a)

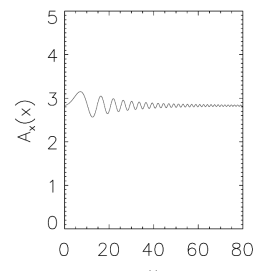

(b)

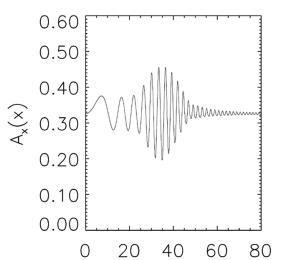

(c)
Fig. 9. Spatially oscillating pump with a positiondependent wave number. $E_{x \text {, mid }}=4, \delta E_{x}=1, x_{m}=80$. (a) Input signal. (b) Effect of diffraction alone. (c) Interplay of diffraction and nonlinearity.
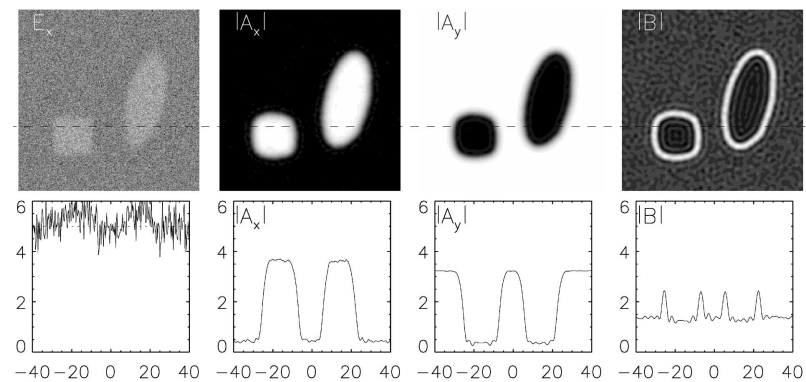

Fig. 10. Contrast enhancement and noise filtering of a noisy image.

input image and sets a minimum contrast below which no contrast enhancement can occur.

Finally, the more realistic case of a noisy image was considered by superposing a static random field distribution on the previous image. In the frequencyconversion regime (Fig. 8) both the upconverted image at the $\mathrm{SH}$ frequency and the transmitted image at the fundamental frequency have a lower noise level than the input image. The underlying noise-filtering mechanism results from the interplay of diffraction and nonlinearity, which is best illustrated by considering a one-dimensional image,

$$
E_{x}(x)=E_{x, 0}+\delta E_{x} \cos \left(k_{m} \frac{x^{2}}{2 x_{m}}\right),
$$

corresponding to an oscillatory perturbation of a homogeneous field, with a wave number that increases linearly with the position $x$ [Fig. 9(a)]. If we consider diffraction alone $\left[E_{y}=0\right.$ in Eqs. (1)-(3)], $A_{x}$ satisfies the linear equation

$$
\partial_{t} A_{x}=-(1+i \Delta) A_{x}+i \nabla_{\perp}^{2} A_{x}+E_{x},
$$

and the noise is transmitted mainly around the zero spatial frequency. Therefore the amplitude of the oscillations in $A_{x}(x)$, for the input image described by Eq. (5), decreases with the position $x$ [Fig. 9(b)]. By activating the nonlinear interaction by injecting a $y$-polarized pump field $E_{y}=5$, the shape of the envelope of the oscillations [Fig. 9(c)] reveals a maximum transmission at a characteristic finite wave number, which sets the typical spatial scale of the labyrinthic pattern observed as a noisy background in Fig. 8. Hence type II SHG acts as a spatial bandpass filter, and applications for contrast inversion, in analogy with the optical parametric amplifier, ${ }^{1}$ could be envisaged.

The noise-filtering effect is more effective in terms of the signal-to-noise ratio when operating in the contrast enhancement regime. As shown in Fig. 10, whereas the objects are difficult to distinguish in the input image, they appear with high contrast at both polarizations (in negative at polarization $y$ ), and at the scale of observation the noise has been washed out almost completely. At the SH frequency the contour of the objects appears clearly, with a slightly noisy background.

This study has shown that, by injecting an optical image in one polarization and a homogeneous field in the orthogonal polarization, according to their relative strength, it is possible to either transfer the image to the SH frequency or enhance its contrast and image its contour. The latter regime should be useful for regenerating a weak image deteriorated by a strong noisy background. In both regimes interesting noisefiltering effects were demonstrated. Our theoretical calculation seems to be within accessible values of current SHG experiments. ${ }^{11}$ For example, with a 1-cmlong $\mathrm{KNbO}_{3}$ crystal, ${ }^{11}$ the typical pump field $E_{x}=$ $E_{y}=5$ corresponds to an intensity $I=860 \mathrm{~kW} / \mathrm{cm}^{2}$, yielding a power of $P=1.56 \mathrm{~W}$. Therefore we hope that our calculations stimulate experimental efforts in applications of nonlinear optics for all-optical image processing.

P. Scotto's e-mail address is pierre@imedea.uib.es.

\section{References}

1. F. Devaux and E. Lantz, Opt. Commun. 114, 295 (1995).

2. M. Y. Shih, A. Shishido, and I. C. Khoo, Opt. Lett. 26, 1140 (2001).

3. J. Khoury, C. L. Woods, and M. Cronin-Golomb, Opt. Lett. 16, 747 (1991).

4. J. Khoury, J. Fu, M. Cronin-Golomb, and C. Woods, J. Opt. Soc. Am. B 11, 1960 (1994).

5. U. Peschel, C. Etrich, and F. Lederer, Opt. Lett. 23, 500 (1998).

6. U. Peschel, C. Etrich, and F. Lederer, Phys. Rev. E 58, 4005 (1998).

7. S. Longhi, Opt. Lett. 23, 346 (1998).

8. S. Longhi, Phys. Rev. A 59, 4021 (1999).

9. For homogeneous pumps the pumping amplitudes can be taken as real fields without loss of generality.

10. Z. Y. Ou, Phys. Rev. A 49, 4902 (1994).

11. M. Bache, P. Lodahl, A. V. Mamaev, M. Marcus, and M. Saffman, Phys. Rev. A 65, 033811 (2002). 